\title{
Point defects in GaAs photonic crystals
}

\author{
S. E. Dissanayake, K. A. I. L. Wijewardena Gamalath \\ Department of Physics, University of Colombo, Colombo 3, Sri Lanka \\ E-mail address: imalie@phys.cmb.ac.lk
}

\begin{abstract}
Applying plane wave expansion method to one dimensional multilayer system formed from alternating layers of GaAs and air, a defect mode was artificially introduced by removing a GaAs layer at the centre of a supercell and the band structures and mode field distributions were obtained. The defect mode normalized frequency was 0.28 . The parameters for developing a Febry-Perot filter in the visible frequencies are given. The point defects in a two dimensional square lattice formed from GaAs rods of radius $0.16 a$ in air was created by removing one GaAs rod at the centre of a $5 \times 5$ supercell and also by increasing the centre GaAs rod radius to $0.5 \mathrm{a}$. The removal of the rod folded the band structure 25 times creating a localized evanescent defect mode. The increment of the centre GaAs rod radius led to five defect bands inside the band gap with one degenerate state creating a monopole, two quadrupoles and two hexapoles. Evolution of the defect modes with the variation of the radius of GaAs rod is also presented.
\end{abstract}

Keywords: Photonic crystal, square lattice, plane wave expansion, GaAs, multilayer, supercell, band structure, mode field distribution, defect mode.

\section{INTRODUCTION}

Photonic crystals tailor the propagation of electromagnetic waves in a unique way. Electromagnetic (EM) waves propagating through these artificially arranged periodic structures with a periodically modulated dielectric constant are organized into photonic bands separated by gaps, within which the propagating states are forbidden. Maxwell's Equations and Bloch theorem are predominant in the study of photonic crystals. Since they are high in precision, the properties of photonic materials are highly predictable and controllable. In electronic crystals, properties are governed by Schrödinger equation and subjected to diffraction of unnecessary defects. But in photonic crystals, the defects are added to localize electromagnetic states or propagating states on purpose. The formation of photonic band gaps in photonic materials is a result of macroscopic Bragg scattering related to periodicity or the geometry of the lattice and the microscopic Mie scattering related to the shape of individual scatters. The main parameter that affects the strength of these two scattering mechanisms is the refractive index contrast. The effect is complex and hence there is no simple correlation between the band gap and the parameters of photonic structures. As a consequence, numerical calculations play a vital role in predicting the properties of light in these structures.

One dimensional multilayer system was first studied in 1887 by Lord Rayleigh [1] and a detailed theoretical study was carried out by Bykov [2] speculating the possibility of two or three dimensional periodic optical structures. Engineering the photonic density of states, 
Yablonovitch [3] proposed to control the spontaneous emission of materials embedded within the photonic crystals. Photonic crystals have been used to effect the localisation and the control of light [4]. The existence of complete band gaps in a photonic crystal was correctly predicted by Ho et al.[5]. Among the suggested techniques for calculating photonic band gap properties are the transfer matrix and scattering matrix method, plane wave expansion method, finite difference time domain (FDTD) method, method of momentum, finite element method, etc. Many previously unattainable numerical calculations have been successfully solved by means of iterative optimization of an approximate initial solution, through a parallel computing approach via block matrix diagonalization [6]. The plane wave expansion method has been adopted to compute the diffraction of two-dimensional periodic bandgap materials with a finite thickness [7].

In the present work, the plane wave expansion method was used to study the one dimensional multilayer photonic system formed from alternating layers of GaAs of thickness $0.3 a$ and air. Using the supercell concept, a defect mode was artificially created by removing a GaAs layer at the centre of a supercell. For the development of a Febry-Perot filter in the visible frequencies, the parameters are presented. For a two dimensional square lattices formed from the circular cylindrical GaAs rods of radius $0.16 a$ in air, a point defects was created by two methods, namely removing the GaAs rod at the centre of a $5 \times 5$ supercell and by increasing the centre GaAs rod radius to $0.5 \mathrm{a}$. The band structures and mode field distributions for all these cases are presented. The defect mode frequency was tuned by varying the defect radius of the GaAs rod.

\section{EIGENMODES AND EIGENFREQUENCIES}

The propagation of light in a photonic crystal is governed by the macroscopic Maxwell equations. For the propagation of electromagnetic waves in a macroscopic, homogeneous, isotropic dielectric material free of charge sources (i.e. $\rho=0, \boldsymbol{J}=0$ ) with real dielectric constant $\varepsilon(\boldsymbol{r})$ and no dispersion, lead to a master equation in magnetic field $\boldsymbol{H}(\boldsymbol{r})$ [2]:

$$
\nabla \times \frac{1}{\varepsilon(\boldsymbol{r})} \nabla \times \boldsymbol{H}=\frac{\omega^{2}}{c^{2}} \boldsymbol{H}(\boldsymbol{r})
$$

The wave propagation in three-dimensional periodic media is capable of propagating without scattering and the Bloch's theorem state that the behaviour is governed by a periodic envelope function $u(\boldsymbol{r})$ multiplied by a plane wave [8]. Therefore the magnetic field $\boldsymbol{H}$ can be expressed as,

$$
\boldsymbol{H}(\boldsymbol{r})=e^{i \boldsymbol{k} \cdot \boldsymbol{r}} u(\boldsymbol{r}) \hat{e}_{k} ; \quad u(\boldsymbol{r})=u(\boldsymbol{r}+\boldsymbol{R})
$$

where $\boldsymbol{R}$ is an arbitrary lattice vector and $\hat{e}_{k}$ is a unit vector perpendicular to the wave vector $\boldsymbol{k}$ and parallel to the magnetic field vector $\boldsymbol{H}$ reflecting its transversal property. The periodic dielectric function can be Fourier transform to reciprocal space, giving an arbitrary spatial frequency call reciprocal lattice vector $\boldsymbol{G}_{i}=h_{i} \boldsymbol{b}_{i}$ in terms of basis vectors in the reciprocal space $\boldsymbol{b}_{i}$ and set of are integers $h_{i}$, 
$\varepsilon(\boldsymbol{r})=\sum_{G_{i}} \varepsilon\left(\boldsymbol{G}_{i}\right) e^{i \boldsymbol{G} \cdot \boldsymbol{r}}, \quad \varepsilon(\boldsymbol{G})=\int_{A} \varepsilon(\boldsymbol{r}) e^{-i \boldsymbol{G} \cdot \boldsymbol{r}} d^{2} r$

The Fourier component $\varepsilon\left(\boldsymbol{G}-\boldsymbol{G}^{\prime}\right)$ is calculated by integrating over the area $A$ of one lattice unit cell. Periodic dielectric function in real space can be simplified. Fourier transformation of the dielectric function can be further simplified to,

$$
\begin{aligned}
\varepsilon(\boldsymbol{G}) & =f \varepsilon_{a}+(1-f) \varepsilon_{b} & & \text { for } \boldsymbol{G}=0 \\
& =\left(\varepsilon_{a}-\varepsilon_{b}\right) S(\boldsymbol{G}) & & \text { for } \boldsymbol{G} \neq 0
\end{aligned}
$$

where $\varepsilon_{a}$ and $\varepsilon_{b}$ refer to the dielectric constants of the localized medium and background respectively and $f$ is the filling factor, defined as the fraction of area occupied by the localized medium in one unit cell. The factor $S(\boldsymbol{G})$ relying on the geometry of the localized medium and the lattice structures is given by,

$$
S(\boldsymbol{G})=\frac{1}{A} \int_{A_{d}} e^{-i \boldsymbol{G} \cdot \boldsymbol{r}} d^{2} r
$$

Since $u(\boldsymbol{r})$ has the same periodic property as $\varepsilon(\boldsymbol{r}), \boldsymbol{H}$ can be expanded as:

$$
\boldsymbol{H}(\boldsymbol{r})=\sum_{\boldsymbol{G}_{i}, \lambda} h\left(\boldsymbol{G}_{i}, \lambda\right) \hat{e}_{\lambda} e^{i\left(\boldsymbol{k}+\boldsymbol{G}_{i}\right) \cdot \boldsymbol{r}}
$$

where $\hat{e}_{\lambda}$ are two orthogonal unit vectors perpendicular to $\boldsymbol{k}+\boldsymbol{G}_{i}$. This leads to standard eigenvalue problem

$$
\sum_{\boldsymbol{G}^{\prime} \lambda^{\prime}} \varepsilon_{\boldsymbol{G}-\boldsymbol{G}^{\prime}}^{-1}\left[\left(\boldsymbol{k}+\boldsymbol{G}^{\prime}\right) \times \hat{e}_{\lambda^{\prime}}\right]\left[(\boldsymbol{k}+\boldsymbol{G}) \cdot \hat{e}_{\lambda}\right] h_{\boldsymbol{G}^{\prime} \lambda^{\prime}}=\frac{\omega^{2}}{c^{2}} h_{\boldsymbol{G} \lambda} .
$$

For every wave vector $\boldsymbol{k}$, the eigenvalue of the above equation gives the frequency $\omega$ of the eigenmode. When $N$ plane waves are used for each $k$ point, there will be $2 N$ number of discrete frequencies. A band is formed by the eigenfrequencies of the same order of the $\boldsymbol{k}$ vectors in the first irreducible Brillouin zone. For transverse electric (TE) wave, the $\boldsymbol{D}$ field is in the x-y plane and the $\boldsymbol{H}$ field is along the $z$-direction is given by

$$
\boldsymbol{H}_{k}^{w}(\boldsymbol{r})=\sum_{G} h_{1}(\boldsymbol{G}) \hat{z} \cdot e^{i(k+G) \cdot r}
$$

For transverse magnetic (TM) wave, the $\boldsymbol{H}$ field is in the x-y plane and the $\boldsymbol{D}$ field along z direction is,

$$
\boldsymbol{D}_{k}^{w}(\boldsymbol{r})=\frac{1}{|\boldsymbol{k}|} \sum_{\boldsymbol{G}}|\boldsymbol{k}+\boldsymbol{G}| h_{1}(\boldsymbol{G}) \cdot e^{i(\boldsymbol{k}+\boldsymbol{G}) \cdot \boldsymbol{r}} \hat{z}
$$


In order to use plane wave expansion method in the calculation of a defect mode, a defect is artificially introduced disrupting the periodicity. For this purpose, the defect mode was considered to be at the centre of a new unit cell formed by several successive rows of patterns. The construction of this supercell was then repeated along the different spatial

directions along which the original photonic crystal was periodically organized. This artificial structure consists of periodic defects separated by regions of perfect photonic crystal. The dimensions of the supercell must be sufficiently large to ensure the same spectral properties as the original arrangement. As long as confined modes do not couple to one another,

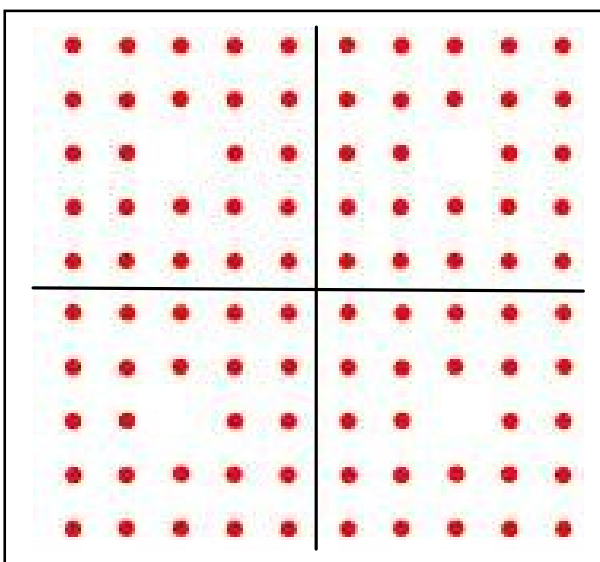

Figure1: $5 \times 5$ super cell then the results of the calculation equally apply to the case of an isolated defect in a large array of perfect photonic crystal. The figure 1 shows a $5 \times 5$ supercell of a square lattice.

\section{POINT DEFECTS IN ONE DIMENSIONAL PHOTONIC CRYSTAL}

A simplest photonic crystal consisting of alternating layers of GaAs of thickness $R$ and air called a multilayer film, shown in figure 2 was considered. This arrangement is widely used in dielectric mirrors and optical filters. The material is periodic in $z$-direction, and homogeneous in the $x y$-plane. The crystal has a continuous translational symmetry in the $x y$ plane, so the wave vector parallel to the plane $\left(\boldsymbol{k}_{/ /}\right)$can assume any value. Since the crystal has a discrete translational symmetry in $z$ direction, the wave vector in $z$-direction $\left(k_{z}\right)$ is restricted to a finite interval, named as one dimensional Brillouin zone $-\pi / a \leq k_{z} \leq \pi / a$.

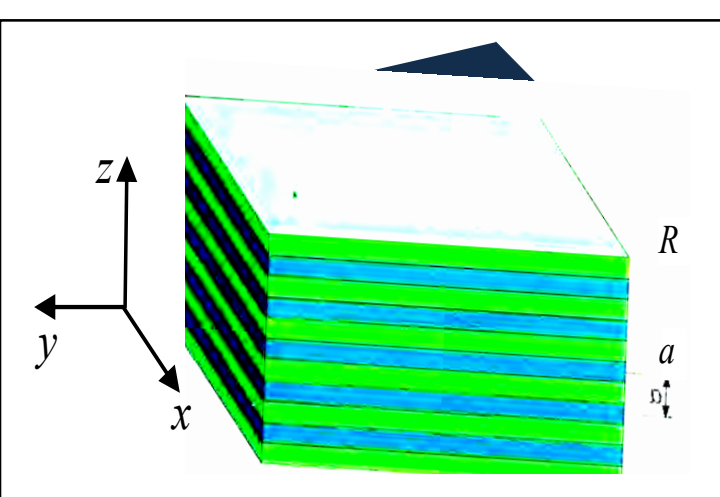

Figure 2: The multilayer film formed from alternating layers of GaAs (dark blue) and air (light blue) spaced by a distance $a$. Film extends indefinitely in the z-direction.

Since $\boldsymbol{k}$ and $\boldsymbol{G}$ vectors have only two possible directions, $+z$ and $-z$, the two unit vectors can be set as $\hat{e}_{1}=\hat{y}$ and $\hat{e}_{2}=\hat{x}$ and the eigenvalues given in equation 7, simplifies to $N$ by $N$ equations: 


$$
\sum_{\boldsymbol{G}^{\prime}}|\boldsymbol{k}+\boldsymbol{G}|\left|\boldsymbol{k}+\boldsymbol{G}^{\prime}\right| \varepsilon^{-1}\left(\boldsymbol{G}-\boldsymbol{G}^{\prime}\right) h\left(\boldsymbol{G}^{\prime}\right)=\frac{\omega^{2}}{c^{2}} h(\boldsymbol{G})
$$

In this case, both displacement field and magnetic field are in $x y$ plane and the transverse electric (TE) and transverse magnetic (TM) modes are similar. Structure factor for one dimensional multilayer system is given by the filling factor $f$ equal to the ratio of the widths of two layers as $f \sin \left(G_{x} a / 2\right) /\left(G_{x} a / 2\right)$. The photonic band structure for a one dimensional multilayer system formed from GaAs layers of thickness $0.3 a$ with a dielectric constant $\varepsilon_{a}=13$ and air layers of thickness $0.7 a$ with dielectric constant $\varepsilon_{b}=1$ is shown in Figure 3. The photonic band gaps occur between the first and second as well as the second and third band frequencies. The displacement field distribution intensity for the states immediately above and below the gap was calculated by considering the eigenvectors and shown in figure 4 at the edge of the Brillouin zone at $k=\pi / a$. The dark

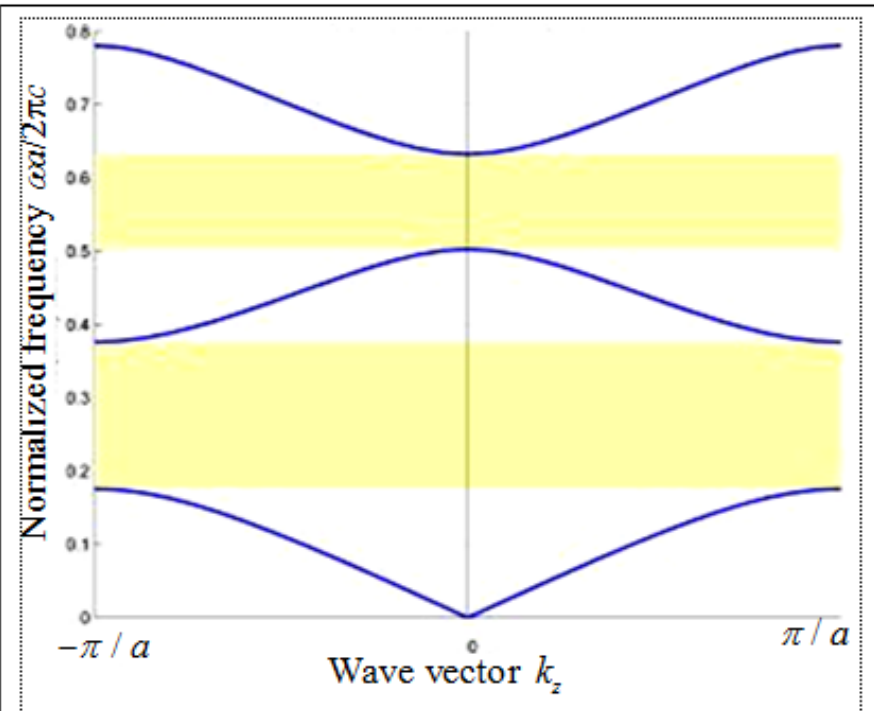

Figure 3: The Photonic band Structure of a multilayer film formed from: altemating dielectric layers of $\mathrm{GaAs}$ of width $0.3 a$ and air layer of width $0.7 a$. ( $a$ is the lattice constant).

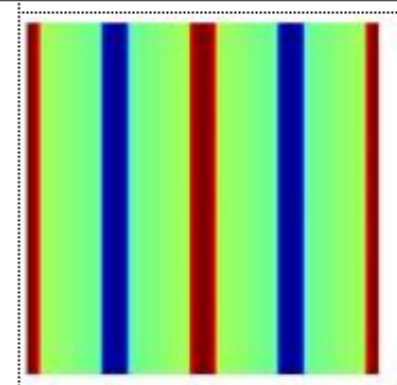

Band 1

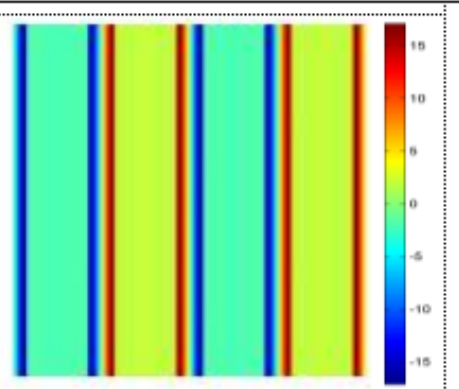

Band 2

Figure 4: The displacement field intensity at $k=\pi / a . R=0.3 a$ red and blue stripes represent GaAs region. The magnitude of the intensity is denoted by the colour variation, red colour denoting a positive field while blue colour denotes a negative field. According to the electromagnetic variational theorem low frequency modes concentrate their energy in the GaAs regions, and the high frequency modes concentrate their energy in the air region. According to the figure 4, modes just under the gap (band 1) have power concentrated in the GaAs region as indicated by strong red and blue stripes, giving it a lower frequency. The modes just above the gap (band 2) have the power concentrated more in air region than in band

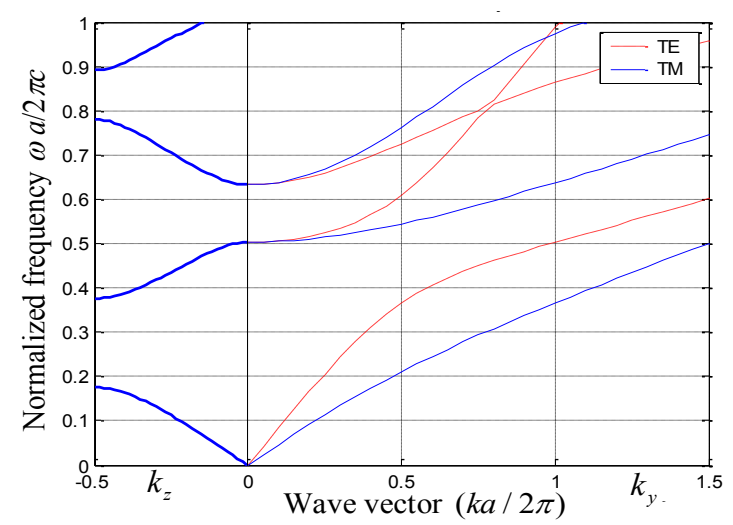

Figure 5: The Band structure of a multilayer film. Left- on axis bands $\left(0,0, k_{z}\right)$. Right - off axis bands $\left(0, k_{y}, 0\right)$ 1, giving rise to a higher frequency. Hence the bands above and below the gap can be categorized by power concentration. The band above the gap is denoted by air band and the 
band below the gap is denoted by dielectric band [6] analogous to the valance and conduction band of a semiconductor crystal.

For the off-axis propagation in one dimensional multilayer system with $k_{x}=0, k_{y} \neq 0, k_{z} \neq 0$, the TM polarized modes and TE-polarized modes are different. From the symmetry, possible polarizations are in the $x$-direction or in the $y z$-plane. $x$-polarized mode is given by equation 10 and $y z$-polarized mode is given by

$\sum_{\boldsymbol{G}^{\prime}}(\boldsymbol{k}+\boldsymbol{G}) \cdot\left(\boldsymbol{k}+\boldsymbol{G}^{\prime}\right) \varepsilon^{-1}\left(\boldsymbol{G}-\boldsymbol{G}^{\prime}\right) h(\boldsymbol{G})=\frac{\omega^{2}}{c^{2}} h(\boldsymbol{G})$

On axis and off axis propagations are shown in the figure 5. There are no band gaps for the off-axis propagation since the off-axis $y$-direction contains no periodic dielectric regions. Since the periodic coupling within the multilayer structure is the origin of the photonic band gaps, its disappearance causes all these photonic band gaps to vanish. Another difference is the degeneracy of the bands. For the on-axis propagation, the electric field is in $x y$-plane. Two polarizations can be selected as the $x$ and $y$-directions. Since they only differ by rotational symmetry, they are degenerated. However for some modes propagating in off axis direction, the degeneracy breakdown since there is no longer the symmetry.

In photonic crystals an artificial disorder can be introduced into the crystal to find localized states in the band gap. These localized states cannot propagate through the material and remain trapped inside a defect. Many applications and devices could be designed using these defects. A point defect in a

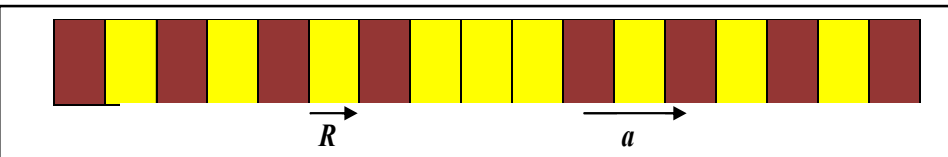

Figure 6: Schematic sketch of the multi layer system with defect introduced by removing GaAs at $\mathrm{z}=0$.

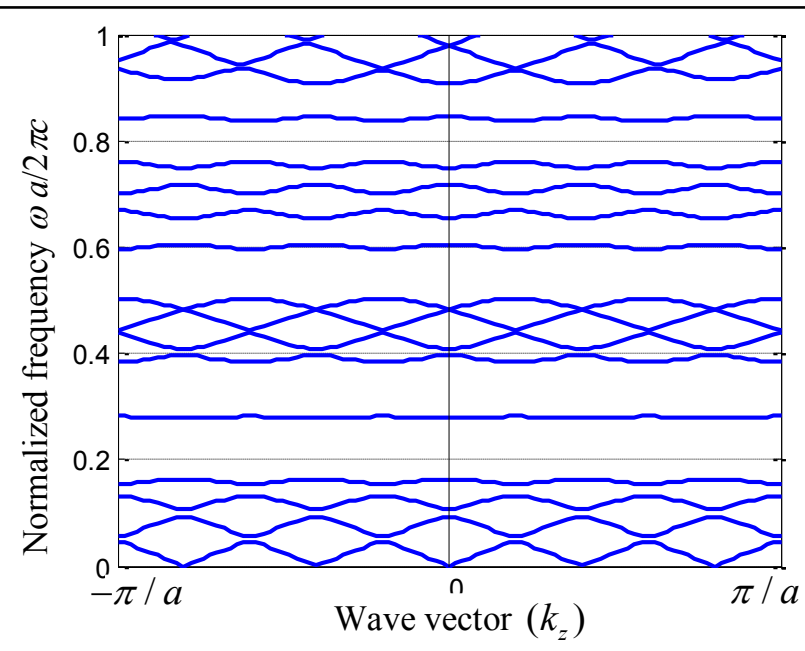

Figure 7: Band diagram of the 1D photonic crystal with the removal of a GaAs layer at $z=0$

one dimensional photonic crystal can be obtained by modifying either the dielectric constant or the thickness of one of the crystal layers equivalent to Febry-Perot cavity. A defect was created by removing a GaAs layer at $z=0$ reducing the dielectric constant of that layer to one. The band structure

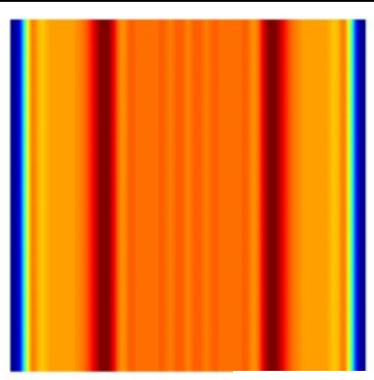

Band 4

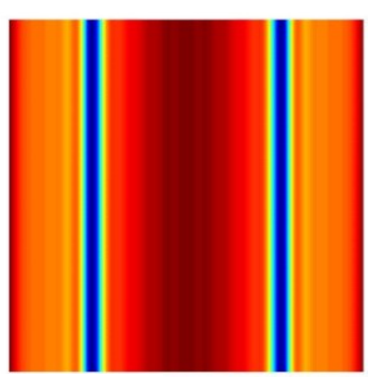

Band 5

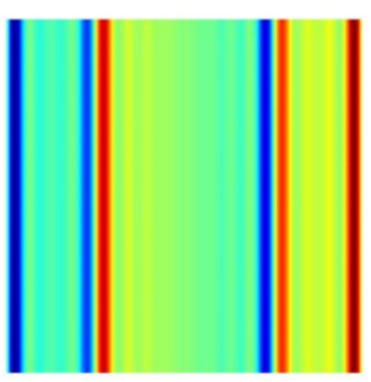

Band 6

Figure 8: Mode field distribution at $k_{z}=\pi / a$ of band 4, 5 and 6 for 1D photonic crystal with the removal of a GaAs layer at $z=0$ 
calculated by the use of a supercell of size 5 is presented in figure 7. For the perfect multilayer system, the first band gap is between the frequencies 0.2 and 0.38 (figure 3). With the introduction of the defect, figure 7 shows an extra mode within the gap with constant $k_{z}$ values. These discrete energy levels, called localized modes are found in the photonic bad gaps. As this mode has a frequency inside the gap, it must decay exponentially once it enters into the crystal. The mode field distribution of bands 4,5 and 6 are shown in figures 8 . The multilayer films on both sides of the defect can act as frequency-specific mirrors. If two such mirrors are kept parallel to one another, then any light propagating in the z-direction will bounce back and forth between these two mirrors and light will be trapped between them. The mode field distribution of band 5 (defect mode) shows a large area in red colour, indicating a strong localization of the energy. It is the defect layer of the crystal.

If a single state can be introduced into the photonic band gap, then the density of states of the system in the band gap is zero, except for a one particular frequency. A band pass filter known as dielectric Febry-Perot filter uses this property, particularly in the visible wavelength range. The defect mode frequency in this case is 0.28133 of normalized frequency $(\omega a / 2 \pi c)$. Filtering wavelength can be adjusted by varying the lattice constant $a$ and keeping the other parameters constant. In order to create a filter in the visible range at a particular wavelength $\lambda$, a multilayer structure with lattice constant $a=\lambda \times 0.2813$ and dielectric layers of $R=0.3 a$ must be used. For example, to create a filter in visible range at wavelength $500 \mathrm{~nm}$, a multilayer structure of lattice constant $140.67 \mathrm{~nm}$ with dielectric layers of thickness is $42.20 \mathrm{~nm}$ is needed. Also the filtering frequency can be adjusted using a different material. But it is always convenient to keep dielectric constant at a fixed value and vary other parameters.

\section{DEFECTS IN TWO DIMENSIONAL PHOTONIC CRYSTALS}

The band gaps of the two dimensional photonic crystals are significantly affected by lattice geometry and the existence of a band gap depends on the lattice to a great extent. The material of the localized medium and the background are also important parameters. The refractive index contrast should be high enough in order to have a considerable band gap. The shape and size of the dielectric rods used to form the photonic crystal also affect the dispersion relationship. The dielectric constants and filling fractions are important parameters and mode field distribution not only depended on geometries but greatly characterized by the cross section of the rods and the shape of the localized medium used [9].

These photonic crystals are periodic along two of its axes and homogeneous along the third. A photonic crystal consisting of a square lattice of circular cylindrical GaAs rods of radius $r$ separated by a lattice constant $a$ in two dimensions is shown in figure 9. The filling fraction $f$, defined as the ratio of volume occupied by rods to the volume of the total space $\left(f=\pi r^{2} / a^{2}\right)$ has a maximum of $f_{\max }=8.54 \%$. Square lattice has two basic lattice vectors $\boldsymbol{a}_{1}=(a, 0,0)$ and $\boldsymbol{a}_{2}=(0, a, 0)$ equal in length and separated by a $90^{\circ}$ angle. The reciprocal lattice points generated by reciprocal lattice basis vectors $\boldsymbol{b}_{1}=(2 \pi / a, 0,0)$ and $\boldsymbol{b}_{2}=(0,2 \pi / a, 0)$, is a square aligned with the direct lattice. The first Brillouin zone is also a square. Figure 10 shows $2 \mathrm{D}$ square lattice, the unit cell with basis lattice vectors $\boldsymbol{a}_{1}$ and $\boldsymbol{a}_{2}$, atom position $(0,0,0) a$ and the Brillouin zone with high symmetry points in irreducible Brillouin zone. 


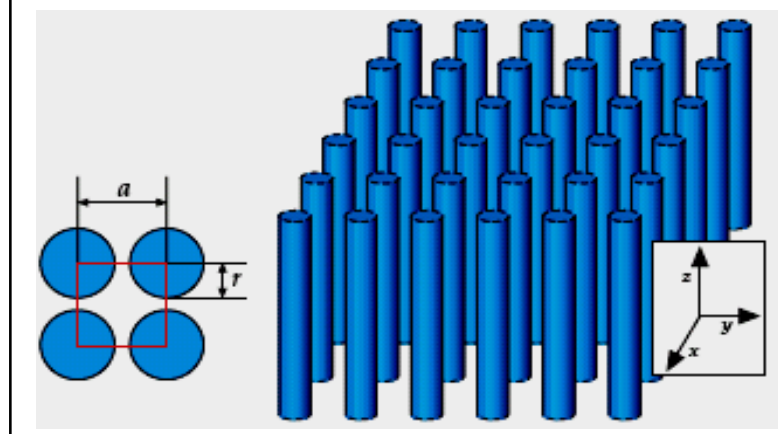

Figure 9: 2D photonic crystal of square lattice formed from cylindrical rods in air

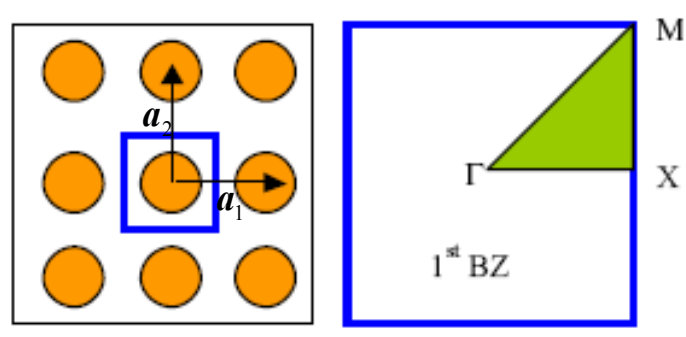

Figure 10: Left- unit cell and basis lattice vectors. Right- Briliouin zone

Although 1D multilayer system only reflects light at normal incidence, two dimensional photonic crystals reflects light incident from any direction in the $x y$ plane. The band structure of a square lattice formed from circular cylindrical GaAs rods of radius $0.16 a$, in air is shown in figure 11 along the edges of the irreducible Brillouin zone, $\Gamma$ to $\mathrm{X}$ and $\Gamma$ to $\mathrm{M}$. A complete band gap was observed between first and second bands in all directions for the TM mode. There is no band gap for TE mode. Even though the filling fraction is small, a large TM band

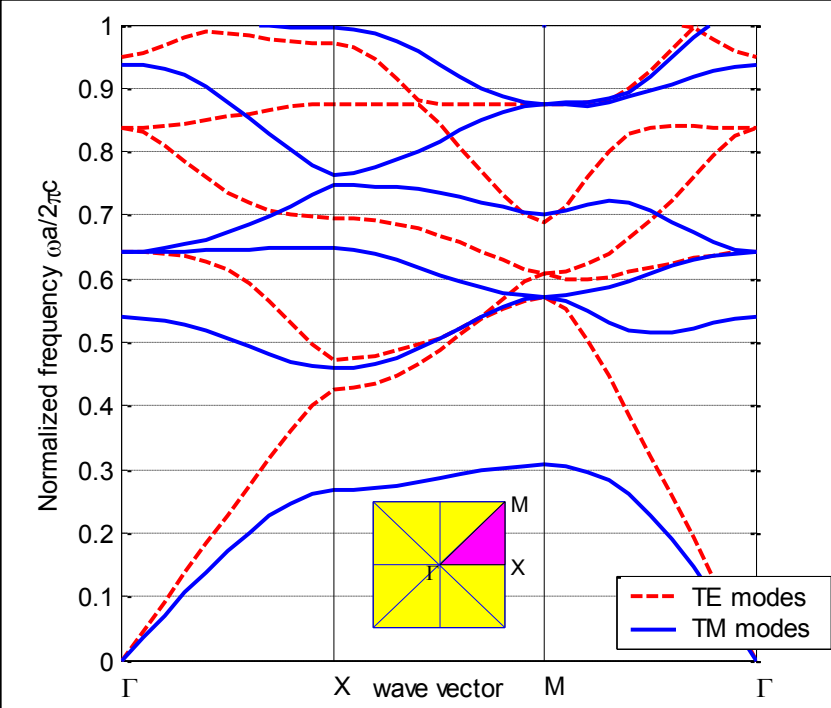

Figure 11: Band structure of a 2D square lattice formed from $\mathrm{GaAs}$ rods of radius $0.16 a$ in air with $f=0.08$

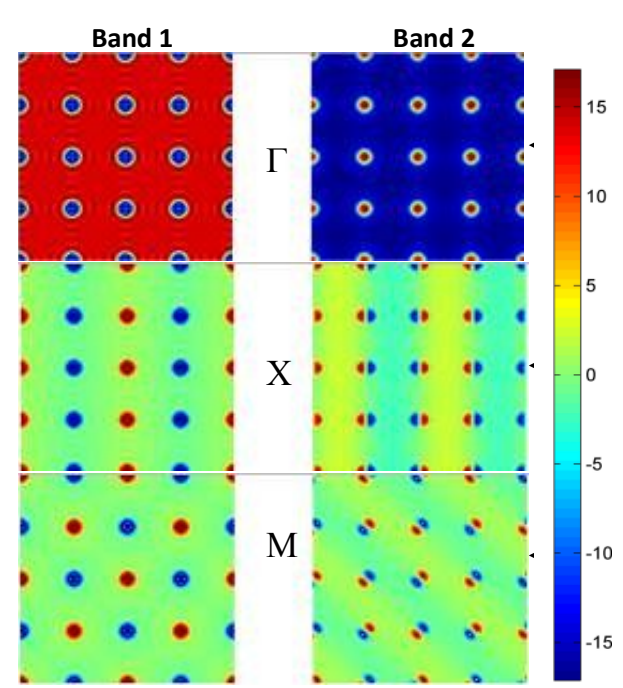

Figure 12: Displacement fields of TM modes for band 1 and band 2

gap is visible around normalized frequency range $0.3069-0.4584$. The displacement field pattern at band 1 and band 2 for $\Gamma, X$ and $M$ points are graphically presented in the figure 12 for TM mode. The band 1 or the dielectric band shows a high energy concentration inside the GaAs region and the field pattern of band 2 or the air band shows that some energy of GaAs regions is expelled into air region. At $X$ and $M$ points, the first mode is a monopole and the second mode is a dipole whereas at $\Gamma$-point both modes are monopoles. 
A point defect in a two dimensional photonic crystals can be introduced by either removing one rod or by increasing the radius of the rod. Former would cause overall dielectric constant to decrease and the latter would increase the overall dielectric constant. Following the variational theorem, if the defect caused the average dielectric constant to decrease, then the eigenfrequencies would shift to higher frequencies, causing one or more bands from the bands near bottom of the band gap to shift in to the band gap. On the contrary, if the defect caused the average dielectric constant to increase, then the eigenfrequencies would shift to lower frequencies, causing one or more bands from the bands near top of the band gap to shift into the band gap. Thus one or more localized modes can be achieved by introducing a point defect.

A point defect was created by removing one GaAs rod from the centre of the $5 \times 5$ supercell (figure 13). The removal of the rod causes the average dielectric constant of the lattice to decrease. The photonic band structure is presented in figure 14 . The band structure has folded 25 times causing the band gap to appear between band 25 and band 26 and the band 25 which was lying previously at the bottom of the band gap has moved into the band gap creating a defect mode. The defect modes are discrete bands, which are constant for all the $k$ vectors in the irreducible Brillouin zone. Since the mode is localized in the band gap, it is evanescent, i.e. the defect mode cannot propagate to the rest of the crystal. This localized mode act as a cavity surrounded by reflecting walls. The mode field distribution of the degenerate bands

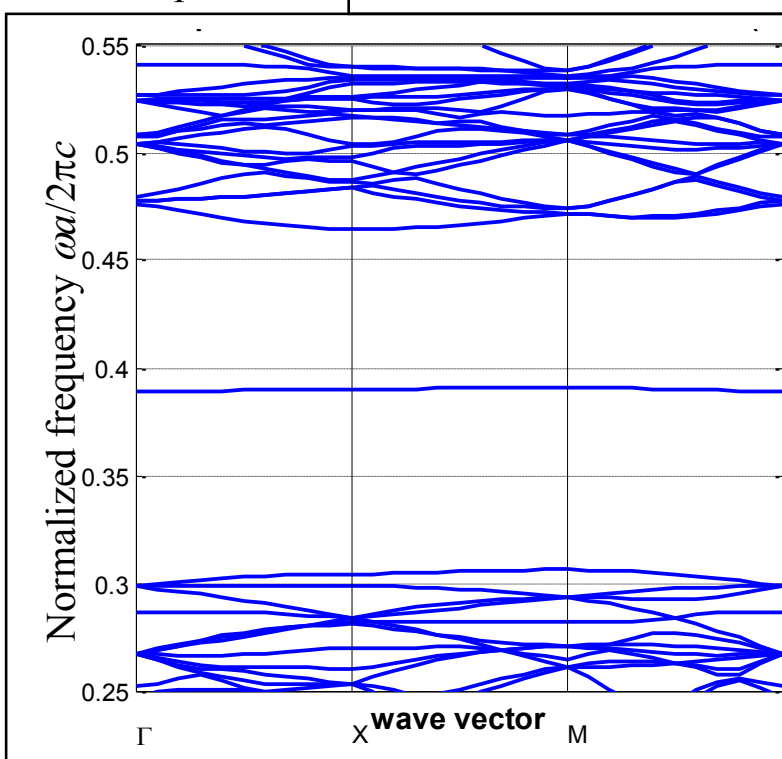

Figure 14: Band structure of a 2D square lattice formed from GaAs rods of radius $0.16 a$ in air with the removal of the GaAs rod at the centre of the supercell $(\mathrm{TM})$

23 and 24, 25 and 26 are shown in figure 15. The band 25 is the defect band and the displacement field intensity is very high, localized at the point defect. Therefore the defect rod acts as a cavity surrounded by reflecting walls. Light cannot escape from the cavity and this concept is very useful in manufacturing resonant cavities for lasers. 
A point defect was also created by increasing the radius of the centre GaAs rod to $0.5 a$ in the $5 \times 5$ super cell (figure 16). The average dielectric constant increased after the defect was introduced, causing the bands above the band gap to shift into the band gap. The band diagram of the crystal with this point defect is shown in figure 17 . The band diagram shows five defect bands inside the band gap with one degenerate state. The defect modes are shifted from the modes above the band gap and lie inside the band gap between the normalized frequencies 0.3069 and 0.45840 . The mode field distribution pattern of the defect bands are shown in figure 18 . The lowest two defect modes, band 28

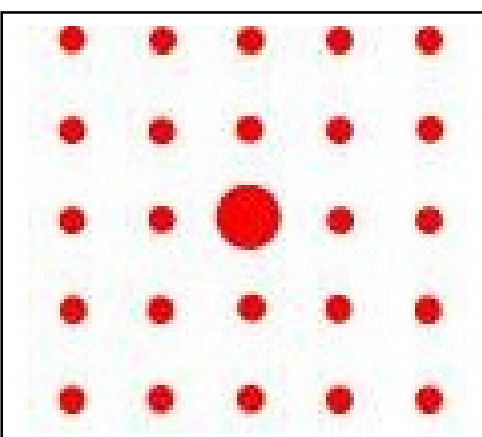

Figure 16: $5 \times 5$ super cell with the increased radius of the centre GaAs rod and band 29 are quadrupoles. The phase difference between each pole alternates. The band 30 is a monopole and the degenerate bands, 31 and 32 form hexapoles with alternating phases. As the mode frequency increases, the number of nodes increases and mode profile becomes more complex. This structure can be used as a multi frequency filter or as a resonant cavity for modes of normalized frequencies $0.4482,0.3658,0.3742,0.3328$. Point defect based micro cavity is very small in size and has an extremely narrow spectral width and high $Q$ values. It can act as a high-Q filter, or a centre of energy transfer. The $Q$ value of the resonant cavity cannot be calculated using plane wave expansion method. FDTD simulation must be done in order to find the $\mathrm{Q}$ value of the resonant cavity.

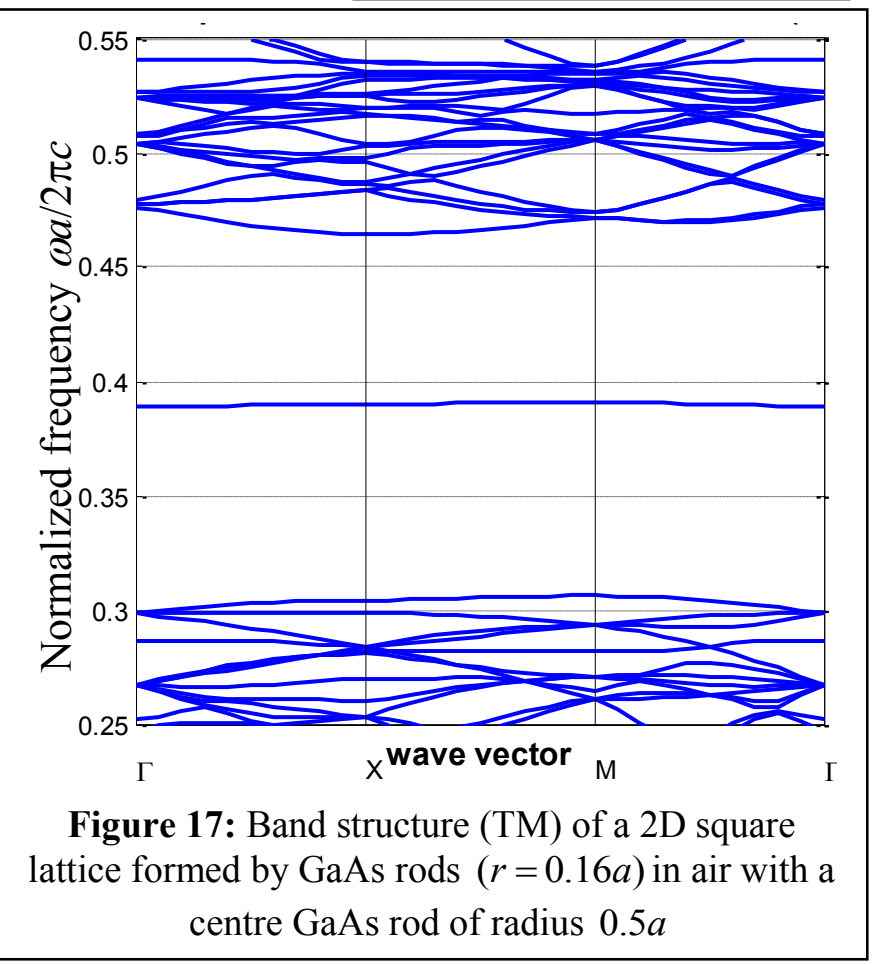

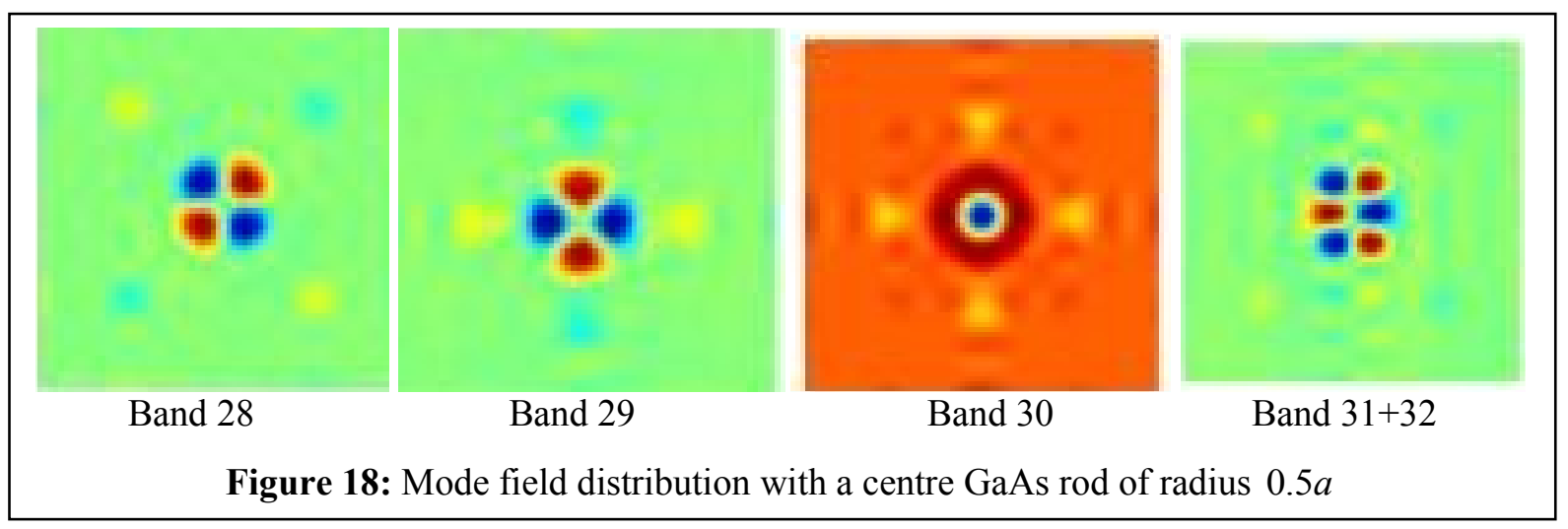


The defect mode frequency can be tuned in several ways. Tuning with varying defect radius is the most efficient method. By selecting the exact frequency of the mode that is evanescent inside the crystal or selecting the frequency of the filter to be constructed, the defect mode frequency can be tuned. Figure 19 shows the evolution of defect modes of a perfect photonic crystal of

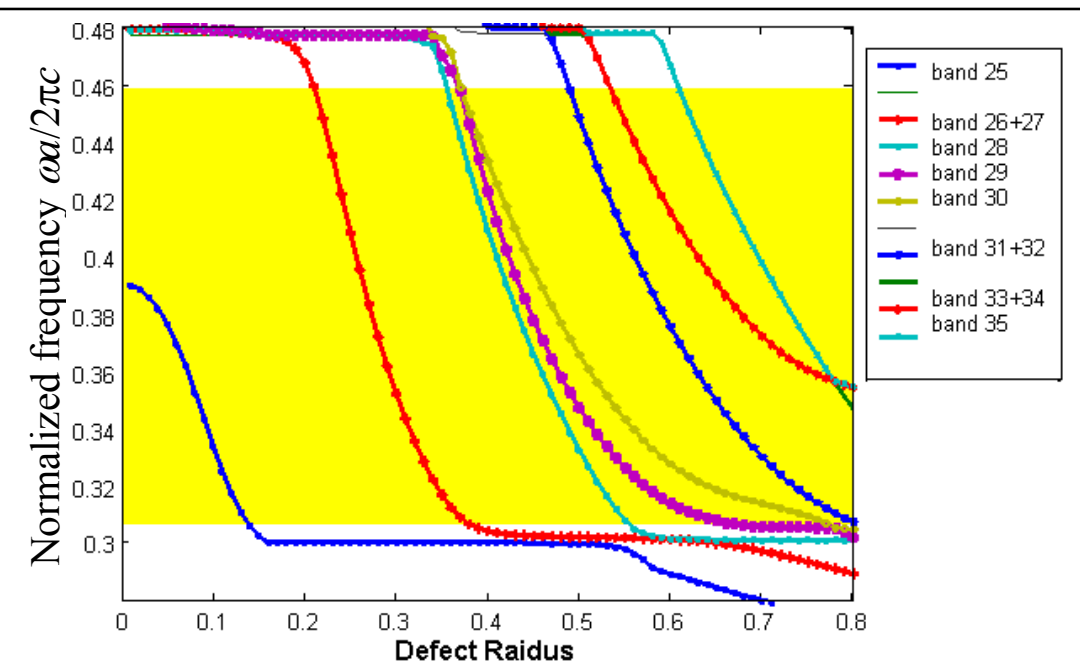

Figure 19: Evolution of the defect modes as the radius of the defected rod at centre of the supercell is increased in a square lattice formed from circular GaAs rod of radius $0.16 a$ in air. square lattice formed from circular GaAs rods in air with a point defect caused by increasing the the radius of the centre GaAs rod of the supercell. For the defect radius of $0.16 a$ representing the perfect square lattice array, no defect mode can be observed in the band gap, which is indicated by yellow. The defect radius of zero represents the point defect introduced by removing a GaAs rod at the centre. By reducing the radius of the rods, only one defect mode can be achieved. As the radius of the defect rod is increased, doubly generate state, band 26 and band 27 shift into the band gap and disappears at the defect radius of $0.4 a$. More and more defect modes start to be formed as radius is increased further and when it equals to $0.63 a$, five defect modes can be observed inside the band gap. This kind of variation is extremely useful in designing resonant cavities or filters as it can be used to find exact conditions to achieve specific defect frequencies. For an example in order to create a single frequency filter operating at normalized frequency 0.4 , a defect GaAs rod with increased radius $0.25 a$ could be used. Another important observation of this diagram is that tuning can be achieved for any frequency in the band gap. The modes follow a path all the way to the bottom of the band gap.

\section{CONCLUSIONS}

Conventional one dimensional multilayer system formed from GaAs layers of thickness $0.3 a$ and air layers of thickness $0.7 a$ was studied by calculating the photonic band structure and mode field distribution pattern using plane wave expansion method. Introducing a point defect by removing one GaAs layer, an evanescent defect mode at normalized frequency 0.28133 was achieved. The value of the parameters needed to create a Febry-Perot filter, within the visible range was evaluated. Plane wave expansion method was very useful and convenient to explore the band structure and mode field pattern of the to dimnsional photonic square crystal formed circular GaAs rods of radius $0.16 a$ in air. A large TM band gap was observed with small filling fractions. The mode field distribution pattern was important to identify the formation of the band gap and symmetries of the system. Point defects were studied for two dimensional square lattices by combining plane wave expansion method and supercell concept. When a GaAs column at the centre of the supercell was removed to create a 
point defect, the displacement field intensity is very high and localized at the point defect. The defect rod acts as a cavity surrounded by reflecting walls and can be used as resonant cavities for lasers. When a point defect was introduced by increasing the radius of the GaAs rod radius at the centre of the supercell to $0.5 a$, five defect modes were observed within the band gap with normalized frequencies in the range $0.3069-0.4584$ giving rise to a monopole, two quadrupoles and two hexapoles. This structure can be used as a multi frequency filter or as a resonant cavity for modes of normalized frequencies $0.4482,0.3658,0.3742,0.3328$. The possibility of tuning the defect mode frequency was provided by calculating the defect mode frequencies for different defect radius.

\section{References}

[1] J. W. S. Rayleigh, Phil. Mag. 26 (1988) 256-265.

[2] V. P. Bykov, Sov. Phys. JETP 35 (1972) 269-273.

[3] E. Yablonovich, Phys. Rev. Lett. 58 (1987) 2059-2062.

[4] S. John, Phys. Rev. Lett. 58 (1987) 2486-2489.

[5] K. M. Ho, C. T Chang, C. M. Soukoulis, Phys. Rev. Lett. 65 (1990) 3152-3155.

[6] S. C. Johnson, J. D. Joannopoulos, Opt. Express 8 (2001) 173-190; J. D. Joannopolus, S.C. Johnson J.N. Winn, R.D. Meade, Photonic Crystals: Modeling the flow of light, Princeton University Press, (2008) Chapter 5, p. 66-72.

[7] K. Skoda, Phys. Rev. B. 52 (1995) 8992-9002.

[8] F. Bloch, Z. Physik 52 (1928) 555-600.

[9] S. E. Dissanayake, K. A. I. L. Wijewardena Gamalath, ILCPA 5 (2014) 58-88. 